\title{
Extended field-of-view microendoscopy through aberration corrected GRIN lenses
}

\author{
Andrea Sattin ${ }^{1}$, Andrea Antonini ${ }^{1,2}$, Serena Bovetti ${ }^{1}$, Claudio Moretti ${ }^{1}$, Angelo Forli ${ }^{1}$, Francesca \\ Succol $^{1}$, Vijayakumar P. Rajamanickam ${ }^{1,2,3}$, Andrea Bertoncini ${ }^{3}$, Carlo Liberale ${ }^{2,3}$, Tommaso \\ Fellin ${ }^{1 *}$ \\ ${ }^{1}$ Optical Approaches to Brain Function Laboratory, Department of Neuroscience and Brain \\ Tecnologies, Istituto Italiano di Tecnologia, Genova, Italy \\ ${ }^{2}$ Nanostructures Department, Istituto Italiano di Tecnologia, Genova, Italy \\ ${ }^{3}$ Biological and Environmental Sciences and Engineering Division (BESE), King Abdullah \\ University of Science and Technology (KAUST), Thuwal 23955-6900, Saudi Arabia
}

\begin{abstract}
Graded index (GRIN) lens-based microendoscopes are widely used to perform two-photon fluorescence microscopy in deep $(>1 \mathrm{~mm})$ regions of highly scattering biological tissue, such as the mammalian brain. However, GRIN microendoscopes are limited by intrinsic aberrations which severely restrict the usable field-of-view (FOV). The effect of aberrations is particularly relevant in ultrathin (diameter $<500 \mathrm{~m}$ ) microendoscopes which allow a less invasive insertion of the optical probe into the brain tissue but which are characterized by relatively small imaging FOV. Currently, there are limited commercially available solutions to correct aberrations in these ultrathin microendoscopes because of the difficulty in fabricating corrective optics at the small spatial scale corresponding to the microendoscope diameter. Here, we report the development and application of a new approach to correct aberrations in GRIN microendoscopes using microfabricated polymeric lenses. Corrective optical elements were first designed using optical simulation software, then fabricated by two-photon lithography, and finally coupled with the GRIN lens to generate aberration-corrected microendoscopic probes. The method that we developed was applied to several types of GRIN lenses that differed in length and diameter, and corrected microendoscopes had up to 9 folds larger FOV compared to uncorrected probes. We put corrected microendoscopes to the test by performing high-resolution functional imaging of hundreds of hippocampal or thalamic cells expressing genetically encoded fluorescent indicators in the mouse brain in vivo.
\end{abstract}

Keywords: endoscopic imaging; two-photon; GRIN; aberration correction; extended field-of-view; cellular resolution.

\section{INTRODUCTION}

Microendoscopes are important tools in neuroscience because they allow morphological and functional investigation of neural circuits in deep brain regions ${ }^{1-5}$. Ideally, microendoscopes should have small radial dimensions and, at the same time, maintain high spatial resolution across a large FOV. This would allow high-resolution population imaging, while minimizing tissue damage. Current microendoscopes are frequently based on GRIN rod lenses which have typical diameter between $0.35-1.5 \mathrm{~mm}$ and are characterized by intrinsic aberrations which significantly reduce the $\mathrm{FOV}^{6}$. This is especially relevant when ultrathin microendoscopes (diameter $\leq 500 \mu \mathrm{m}$ ) are used, because they are characterized by a smaller FOV. Specifically designed optical elements coupled with GRIN rods can be used to correct aberrations ${ }^{3}$. However, technical limitations in manufacturing high-precision optics with small lateral dimensions have so far prevented improvements in the performances of microendoscopes with lateral diameter $<1 \mathrm{~mm}$ using corrective optical microelements 7 .

Here we report the development and application of a new method to correct aberrations and extend the FOV in ultrathin microendoscopes. Extended FOV (eFOV) microendoscopes are composed of a GRIN rod lens (NEM-050-25-10-860-S, purchased from Grintech $\mathrm{GmbH}$, Jena, DE), a glass coverslip and a small diameter $(\leq 0.5 \mathrm{~mm})$ aspheric lens (Figure 1A, B) which was fabricated by two-photon polymerization (TPP) ${ }^{8}$ and plastic molding replication ${ }^{9}$. 
A

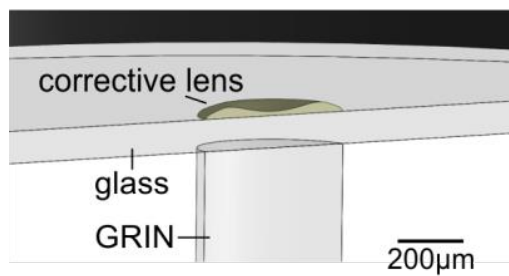

B

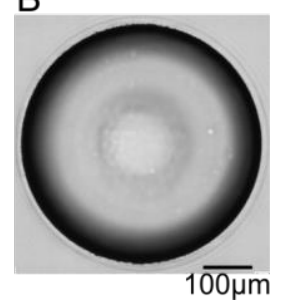

Figure 1. A: schematic of the eFOV-microendoscope mount for head implant. The GRIN rod is attached to one side of the glass coverslip, the microfabricated polymeric lens to the other side of the coverslip. The coverslip is glued on a circular metal ring that facilitates fixation on the animal's skull. B: bright field image (top view) of the aspheric corrective lens.

\section{OPTICAL CHARACTERIZATION}

To characterize the performances of eFOV-microendoscopes, we coupled them with a standard two-photon laser scanning system and we measured the effect of aberration correction on the axial resolution across the FOV. To this aim, we acquired z-stacks of a subresolved thin fluorescent layer (thickness: $300 \mathrm{~nm}$ ) as detailed in ${ }^{10}$. The $\mathrm{x}, \mathrm{z}$ projection of the $\mathrm{z}$-stacks showed higher axial resolution in eFOV-microendoscopes in an area that was $\sim 6$ folds wider compared to uncorrected microendoscopes (Figure 2A, B). Imaging neurons expressing the green fluorescence protein (GFP) in fixed brain slices confirmed the ability of eFOV-microendoscopes to image larger FOV compared to uncorrected endoscopes.
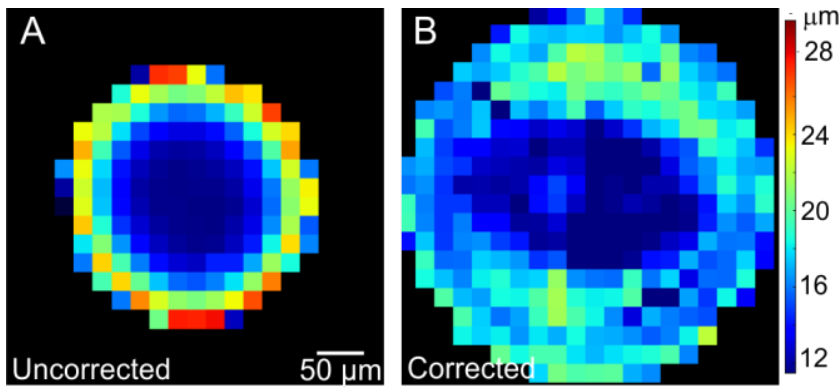

Figure 2. A, B: average axial resolution as a function of the position in the FOV for uncorrected (A) and corrected (B) microendoscopes. The pseudocolor scale indicates axial resolution values in microns. $\mathrm{N}=7$.

\section{IN VIVO APPLICATION}

We expressed the genetically encoded calcium indicator GCaMP6s in the CA1 hippocampal region and the thalamic
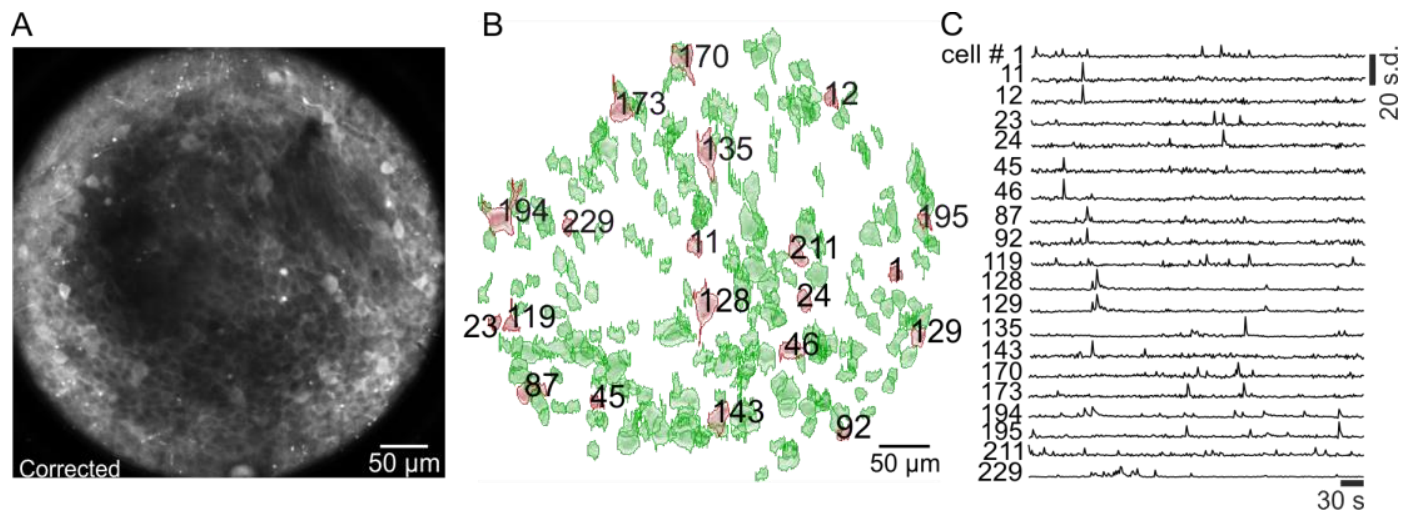

Figure 3. A: two-photon image showing GCaMP6s expressing hippocampal neurons recorded in vivo using eFOVmicroendoscopes. B: identified active ROIs. C: fluorescence signal over time for 20 representative ROIs (red in B). 
ventral posteromedial nucleus and applied eFOV-microendoscopes to perform large scale aberration corrected twophoton functional imaging in vivo. Hundreds of active regions of interest (ROIs) could be imaged on a single plane using ultrathin eFOV-microendoscopes under these experimental conditions (Figure 3), confirming efficient population imaging.

\section{CONCLUSIONS}

In summary, we developed a new methodology to correct aberrations in ultrathin (diameter $<500 \quad \mathrm{~m}$ ) microendoscopes using microfabricated aspherical lenses. eFOV-microendoscopes showed significantly enlarged FOV, allowing efficient population imaging with ultrathin probes. Our approach allows the design of miniaturized optical probes with built-in aberration correction, opening the possibility to use these ready-to-use devices in a large variety of existing optical applications, from brain imaging and manipulation ${ }^{11,12}$ to tissue diagnostic.

\section{REFERENCES}

[1] Jung, J. C. and Schnitzer, M. J., "Multiphoton endoscopy,” Opt. Lett. 28(11), 902-904 (2003).

[2] Flusberg, B. A., Nimmerjahn, A., Cocker, E. D., Mukamel, E. A., Barretto, R. P. J., Ko, T. H., Burns, L. D., Jung, J. C. and Schnitzer, M. J., "High-speed, miniaturized fluorescence microscopy in freely moving mice," Nat. Methods 5(11), 935-938 (2008).

[3] Barretto, R. P. J., Messerschmidt, B., Schnitzer, M. J. and America, N., "In vivo fluorescence imaging with highresolution microlenses," Nat. Methods 6(7), 511-512 (2009).

[4] Resendez, S. L., Jennings, J. H., Ung, R. L., Namboodiri, V. M. K., Zhou, Z. C., Otis, J. M., Nomura, H., Mchenry, J. A., Kosyk, O. and Stuber, G. D., "Visualization of cortical, subcortical and deep brain neural circuit dynamics during naturalistic mammalian behavior with head-mounted microscopes and chronically implanted lenses," Nat. Protoc. 11(3), 566-597 (2016).

[5] Bocarsly, M. E., Jiang, W., Wang, C., Dudman, J. T., Ji, N. and Aponte, Y., "Minimally invasive microendoscopy system for in vivo functional imaging of deep nuclei in the mouse brain," Biomed. Opt. Express 6(11), 223-228 (2015).

[6] Wang, C. and Ji, N., "Characterization and improvement of three-dimensional imaging performance of GRINlens-based two-photon fluorescence endomicroscopes with adaptive optics," Opt. Express 21(22), 27142-27154 (2013).

[7] Matz, G., Messerschmidt, H. and Gross, H., "Improved chromatical and field correction of high-NA GRIN-based endomicroscopic imaging systems for new biophotonics applications," Proc. SPIE 9304, 93041E (2015).

[8] Liberale, C., Cojoc, G., Candeloro, P., Das, G., Gentile, F., De Angelis, F. and Di Fabrizio, E., "Micro-optics fabrication on top of optical fibers using two-photon lithography," IEEE Photonics Technol. Lett. 22(7), 474- 476 (2010).

[9] Schaap, A. and Bellouard, Y., "Molding topologically-complex 3D polymer microstructures from femtosecond laser machined glass," Opt. Mater. Express 3(9), 1428-1437 (2013).

[10] Antonini, A., Liberale, C. and Fellin, T., "Fluorescent layers for characterization of sectioning microscopy with coverslip-uncorrected and water immersion objectives," Opt. Express 22(12), 14293-14304 (2014).

[11] Moretti, C., Antonini, A., Bovetti, S., Liberale, C. and Fellin, T., "Scanless functional imaging of hippocampal networks using patterned two-photon illumination through GRIN lenses," Biomed. Opt. Express 7(10), 3958-3967 (2016).

[12] Forli, A., Vecchia, D., Binini, N., Succol, F., Bovetti, S., Moretti, C., Nespoli, F., Mahn, M., Baker, C. A., Bolton, M. M., Yizhar, O. and Fellin, T., "Two-Photon Bidirectional Control and Imaging of Neuronal Excitability with High Spatial Resolution In Vivo," Cell Rep. 22(11), 2809-2817 (2018). 